\title{
TECCIENCIA
}

\section{Distribution of mass of the protoplanetary disk HL Tau}

\author{
Distribución de Masa del Disco Protoplanetario de HL Tau \\ Nidia Yiseth Buitrago Carreño ${ }^{1}$, Nicanor Poveda Tejada ${ }^{1 *}$, \\ Nelson Vera-Villamizar ${ }^{1}$

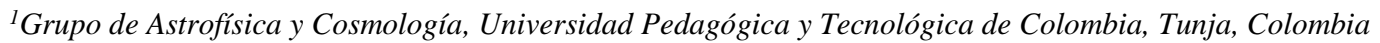

\begin{abstract}
A recent image of ALMA, of the protoplanetary disk around the star HL Tau, shows that the disc consists of several bright concentric rings separated by gaps. There is a hypothesis that these gaps are due to the presence of planets, but there are some difficulties with this interpretation: The planets are too close, generating orbital instability and should not exist planetary formation processes so advanced because the star is too young. This paper shows that the gaps are an effect of the distribution of matter of the disk. In most models assume that the disc consists of a continuous distribution of materia and gas, however, by orbital resonance effect, the disc has an internal structure: It is a superposition of distribution functions that give rise to the characteristic shape of the disc. The mass distribution is obtained, and a preliminary study of the orbits is done, finding that they are stable.
\end{abstract}

Keywords: Solar and Extrasolar System, Orbital Dynamics

\section{Resumen}

Una imagen reciente de ALMA del disco protoplanetario que rodea a la estrella HL Tau, muestra que el disco está formado por varios anillos concéntricos luminosos, separados por gaps. Existe la hipótesis de que estos gaps se deben a la presencia de planetas, pero existen algunas dificultades con esta interpretación: Los planetas se encuentran demasiado cerca, generando inestabilidad orbital, y no deberían existir procesos de formación planetaria tan avanzados, debido a que la estrella es demasiado joven. En este trabajo se muestra que los gaps son un efecto de la distribución de materia del disco. En la mayoría de modelos se asume que el disco consiste de una distribución continua de materia y gas, sin embargo, por efecto de resonancia orbital, el disco tiene una estructura interna: es una superposición de funciones de distribución que dan origen a la forma característica del disco. Se obtiene la distribución de masa, y se hace un estudio preliminar de las órbitas, encontrando que son estables.

Palabras clave: Sistema Solar y Extrasolares, Dinámica Orbital

\section{Introduction}

In 2014 the ALMA (Atacama Large Millimeter/Submillimeter Array) team showed a picture (Figure 1) of the star HL Tau (J2000 04:31:38.45 18:13:9.0), product of continuous emission in the band 6 (211-275 $\mathrm{GHz})$.

*Corresponding Author.

E-mail: nicanor.poveda@uptc.edu.co
In the image several concentric rings appear: rings of gas and dust, emitting radiation which are separated by a gap (dark area), which is due to the existence of a planet, massive enough, to capture the matter found around [1].

How to cite: Buitrago, N., Poveda, N., Vera-Villamizar, N., Distribution of mass of the protoplanetary disk HL Tau, TECCIENCIA, Vol. 11 No. 20, 67-70, 2016, DOI: http:/dx.doi.org/ 
But there are difficulties with this interpretation, since the existence of gaps involves orbital resonance phenomena, which lead to stable orbits, for example, it suffices that two planets are at a greater distance to $3.5 \mathrm{RH}$ (Hill's radii) [2] without however, the gaps are too close to each other, which implies interaction of planets each other, generating an orbital instability which would destroy the observed gaps.

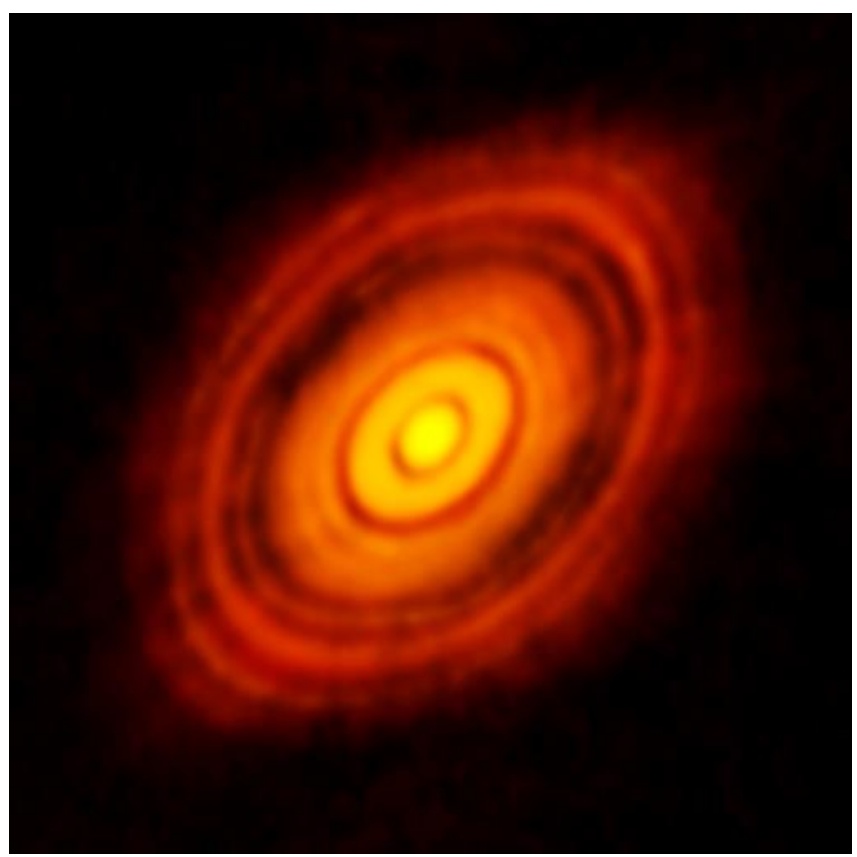

Figure 1 Protoplanetary disk around the star HL-Tau (ALMA)

An attempt was made to explain the existence of stable orbits for planets very close, with the help of the protoplanetary disk []] , but without the orbital migration process eventually the system becomes unstable; the simulations do not show how this situation could occur. Additionally, there should be no planetary formation processes so advanced, because the star is very young, implying that all models of planetary formation are wrong.

In most models it is considered that the protoplanetary disk is formed by a distribution of continuous material, which as it rotates around the star, acquires dynamics (stable and / or turbulent), so that originates planets through a process of accretion of planetesimals.

It is proposed that the protoplanetary disk has a defined internal structure, which gives rise to rings and gaps observed in HL-Tau system and other protoplanetary systems The internal structure originates from the existence of resonance phenomena orbital of the system, leading to a series of overlapping concentric toroids, which shapes the disc. This paper shows how to decompose the protoplanetary disk in this series of toroids, explain the observed distribution of matter, and how it gives rise to a stable planetary system.

\section{Protoplanetary disk model}

The disc matter distribution along the radius $0<\mathrm{r} \leq \mathrm{Ro}$ (AU), gives rise to the ring structure by a superposition of $R_{n}(r) \Theta(\theta)$ basis functions given by:

$$
\rho(r, \theta)=\sum_{n} m_{n} R_{n}(r) \Theta(\theta)
$$

where $\mathrm{n}$ is the number of stable and closed orbits, and $m_{n}$ the mass contained in each element. The radial distribution of matter is described in [4],

$R_{n}(r)=\left[\left(\frac{2}{n a_{s}}\right)^{2 n+1} \frac{1}{(2 n) !}\right] r^{2 n} e^{-2 n / n a_{s}}$

being $a_{s}=k a_{\odot}$, a parameter, $k=M_{s} / M_{\odot}=0,55_{[5]}$ and $a_{\odot}=0,0292705$ [6] see Figure 2(a). The zenithal distribution, Figure 2(b), is:

$$
\Theta_{n}(\theta)=2 \pi\left|Y_{n-1}^{n-1}(\theta, \phi)\right|^{2} \sin \theta
$$

\subsection{Photographic treatment}

The estimated radius of protoplanetary disk of HL Tau, the image from ALMA (ESO/NAOJ/NRAO) is $\mathrm{R}_{0}=117.5 \mathrm{AU}$ with a total mass $\mathrm{M}_{0}=0.135 M_{\odot}$. Initially image of $1800 \times 1800$ pixels is transformed to grayscale; $0^{\circ} \leq \phi<360^{\circ}$ sweep was made.

The major and minor semi-axes of the ellipse were determined by the minimum points, which are $\phi=45.26^{\circ}$, $134.65^{\circ}, 225.17^{\circ}, 315,13^{\circ}$; it is known that the separation should be $\mathrm{n}=(1,3,5,7)$ times 45 , by $\phi / \mathrm{n}$ ratio obtain the position angle photography: $45.04898 \pm 0.07806$, giving rise to an image of $2546 \times 2546$ pixels. Using the expression for ellipticity $\cos (\varphi)=\mathrm{b} / \mathrm{a}$, by minimum sweep the average inclination angle of $\varphi=46.82687^{\circ}$ established; finally an image of $1622 \times 1617$ pixels, obtained (Figure 3.) 


\section{TECCIENCLA}
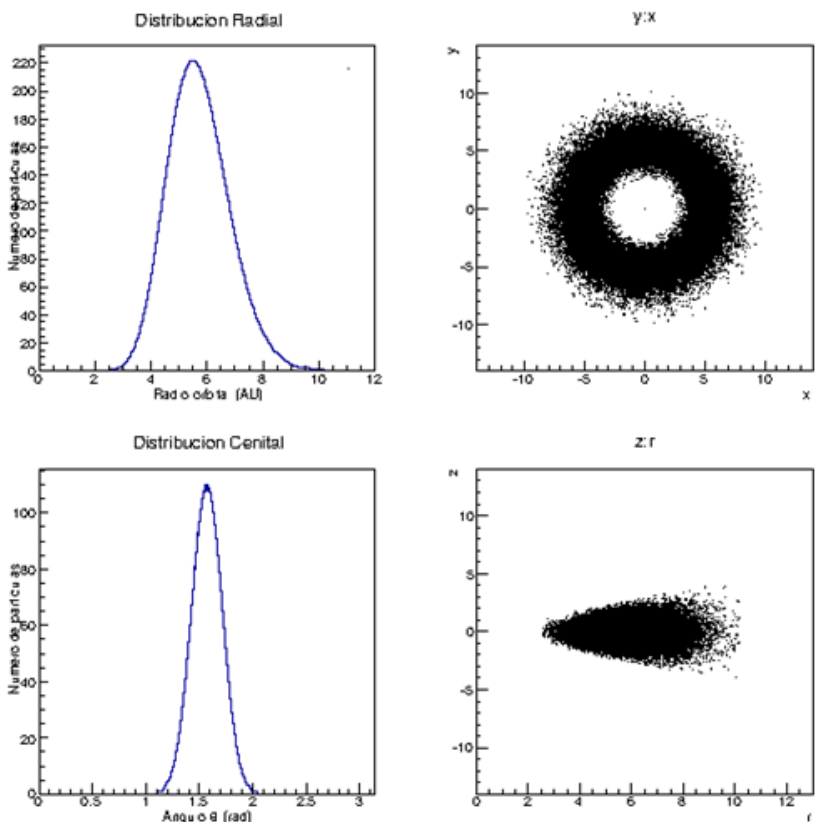

Figure 2 (a) Radial distribution of matter and (b) zenithal distribution.

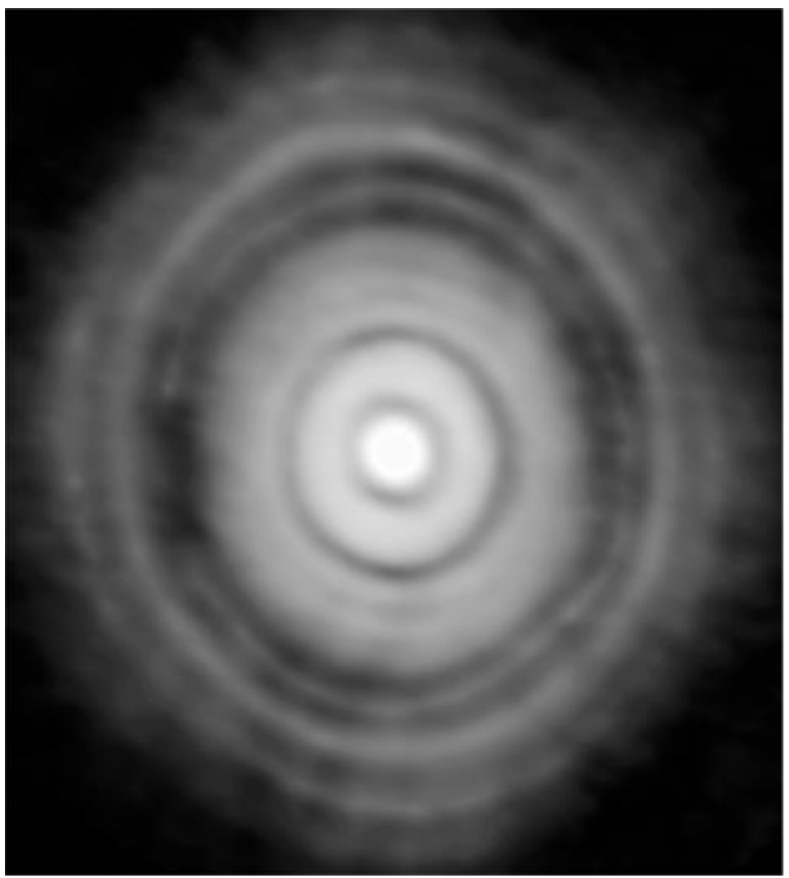

Figure 3 Protoplanetary disk projected image.

In the image a sweep of $0^{\circ} \leq \phi<360^{\circ}$ is made, for each $0<\mathrm{R} \leq \mathrm{R}_{0}$ (AU), obtaining a profile of luminosity-distance, Figure 4 .

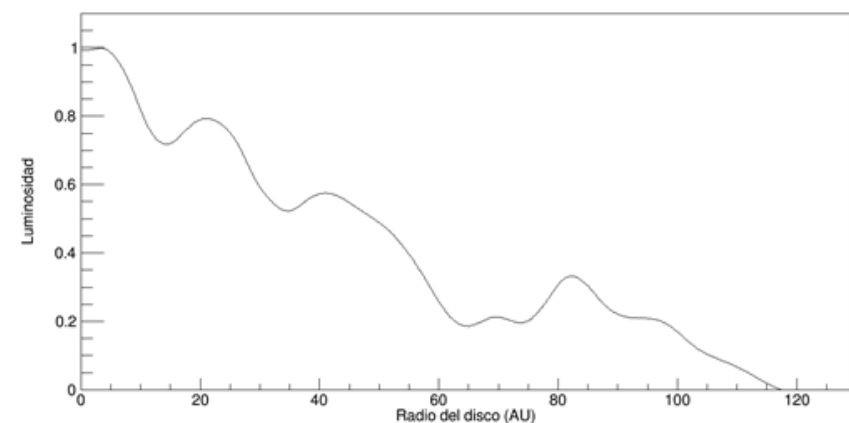

Figure 4 Profile normalized light-distance.

To suppress the effect of brightening the gas due to the presence of the star, a brightness-density conversion is made by the relationship $\rho \sim \mathrm{L}^{3,5}-\exp (-\mathrm{r} / 12.84842)+0.036446$, which depends on the variation of temperature $\mathrm{T} \sim \mathrm{r}^{-0.43}$, obtaining the distribution of matter, $\rho(r, \pi / 2)$ given by equation (1); the area under the curve corresponds to the mass of the disk, Figure 5.

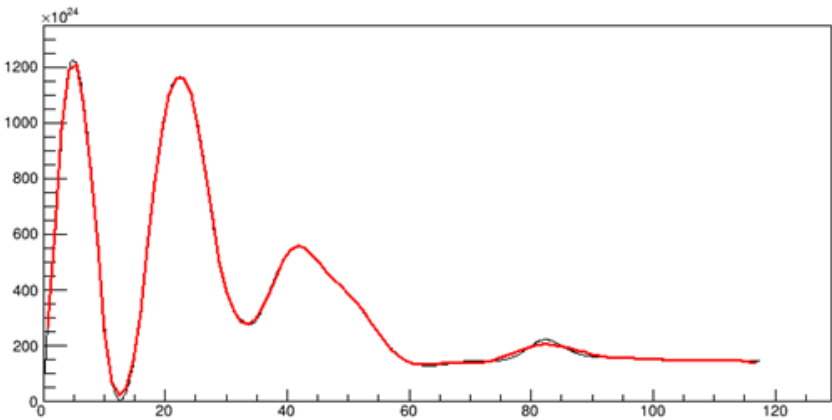

Figure 5: Distance density profile.

An adjustment is made by least squares to obtain the terms of mass $m_{n}$ in equation (1). The red line corresponds to the setting and the masses obtained a computer simulation using the algorithm of Barnes-hut with the integrator IAS15 of rebound [7] develops, is that the orbits are stable for extremely long time, in the Figure 6 orbits and the Hill's radius of objects shown. The main objects are at 17.07, $20.77,41.76,75.98,101.33$ with mass $\geq 1 \mathrm{M}_{\mathrm{J}}$. 


\section{TECCIENCIA}

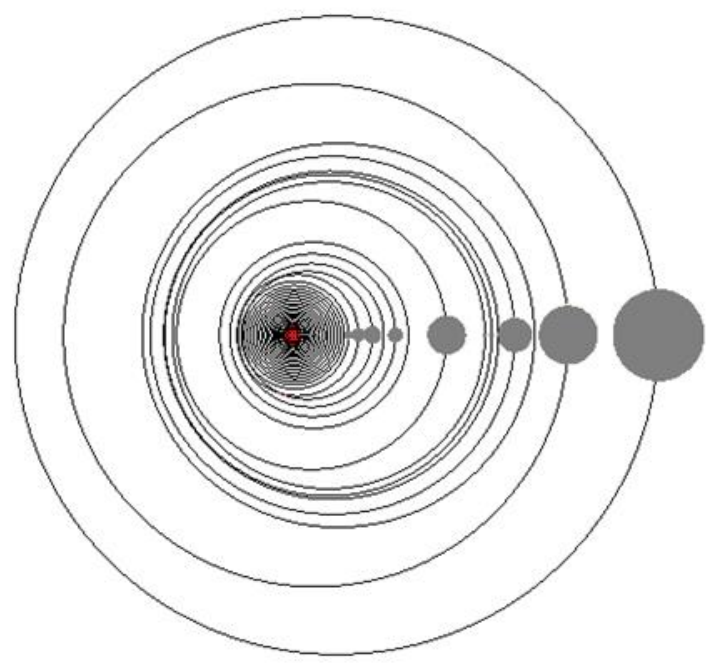

[4]. Nottale L., Schumacher G., and Gay J., "Scale relativity and quantization of the solar system", Astron. Astrophys. 322, 1018-1025, 1997.

[5]. Beckwith, S. V. W. Sargent, A. I., Chini, R. S., \& Guesten, R. ApJ, 99, 924; Sargent, A. I. \& Beckwith, S. V. W. 1990, ApJ, 382, L31, 1990.

[6]. Poveda N., Vera-Villamizar N., Buitrago N. Y., Planetary systems based on a quantum-like model, arXiv:1501.04562.

[7]. Rein, H., Spiegel, D.S., 2015. IAS15: a fast, adaptive, high-order integrator for gravitational dynamics, accurate to machine precision over a billion orbits. MNRAS 446, 1424-1437. 1409.4779.

Figure 6: Orbits and the Hill's radii of the objects

\section{Conclusions}

The observed gaps in the protoplanetary disk HL-Tau are not due to the existence of planets but is an effect of the distribution of matter in the disk, in this model the disc has a discontinuous structure as a series of concentric toroids, rather than a matter continuous distribution. The orbits of the objects obtained are stable.

\section{Acknowledgements}

We thank the Dirección de Investigaciones (DIN) of the Universidad Pedagógica y Tecnológica de Colombia (UPTC).

\section{References}

[1] Kwon W., Looney L. W., Mund L. G., "Resolving the Circumstellar Disk of HL Tauri at Millimeter Wavelengths", ApJ, 741, 1, id. 3, 10 pp., 2011.

[2] Barnes, R., Greenberg, R., "Stability Limits in Extrasolar Planetary Systems", ApJ 647, L163-L166. 2006.

[3] Tamayo D., Triaud A. H. M. J., Menou K., Rein H, "Dynamical Stability of Imaged Planetary Systems in Formation: Application to HL Tau", ApJ, 805,2 , id. 100, 15 pp., 2015.

[3]. Barnes R., Greenberg R., "Stability Limits in Extrasolar Planetary Systems", ApJ 647, L163-L166, 2006. astro-ph/0607210. 